\title{
Synthesis of a conductive copolymer and phase diagram of its suspension with single-walled carbon nanotubes by microfluidic technology
}

Shenghong Yao, ${ }^{\dagger, \ddagger}{ }^{\ddagger}$ Aikaterini Bethani, ${ }^{\dagger}$ Nadia Ziane, ${ }^{\ddagger}$ Cyril Brochon, ${ }^{\dagger}$ Guillaume Fleury, ${ }^{\dagger}$ Georges Hadziioannou, ${ }^{\dagger}$ Philippe Poulin, ${ }^{\S}$ Jean-Baptiste Salmon, ${ }^{\ddagger *}$ Eric Cloutet, ${ }^{\dagger, *}$

${ }^{\dagger}$ Laboratoire de Chimie des Polymères Organiques (LCPO), CNRS-Université de Bordeaux-INP (UMR5629), B8 Allée Geoffroy Saint Hilaire, 33615 Pessac Cedex, France

${ }^{\ddagger}$ Laboratoire du Futur (LOF), CNRS (UMR5258), Université de Bordeaux, 178 Avenue du Docteur Schweitzer, 33608 Pessac Cedex, France

${ }^{\S}$ Centre de Recherche Paul Pascal(CRPP), Université de Bordeaux, 115 avenue Schweitzer, 33600 Pessac Cedex, France 


\begin{abstract}
Amphiphilic block copolymers composed of a poly(3-hexylthiophene) (P3HT) segment and a sulfonated polystyrene (PS-stat-PSS) sequence with well-defined and easily tunable structure were synthesized through Grignard metathesis polymerization (GRIM), RAFT polymerization and sulfonation of PS. Due to the hydrophilic nature and ionic conductivity of the PSS segment, such copolymer shows good solubility in water and high conductivity $\sim 1 \mathrm{~S} / \mathrm{m}$ in form of dry film. Conductivity can be further enhanced with the addition of single-walled nanotubes (SWNTs). The present amphiphilic block copolymer enables efficient unbundling and stabilization of SWNTs in water. With the help of an original microfluidic technique referred to as microfluidic pervaporation, we investigated the concentration process of SWNT/copolymer suspensions up to dry films and obtained a complete phase diagram which reveals the aggregation of SWNT during the concentration process in a given concentration range. High conductivity of about $370 \mathrm{~S} / \mathrm{m}$ is achieved for SWNT/copolymer nanocomposites at high concentrations of SWNTs. The microfluidic pervaporation method is also shown to provide a direct determination of the CNT percolation threshold.
\end{abstract}

Keywords: Carbon nanotube, Conductive inks, Copolymers, Microfluidics, Phase diagram 


\section{Introduction}

Flexible electronics and printed organic electronics are considered nowadays as key technologies in academy and obviously in industry. Their development will be based on the availability of new organic materials which combine deformability and suitable electronic properties. ${ }^{1}$ These new materials include conductive polymers and films of conducting nano-objects such as carbon nanotubes and graphene among others. Such materials are often assembled into films, fibers and coatings via liquid processing which include spin coating, roll to roll coating, ink-jet printing, wet fiber spinning, etc. Achievement of optimal properties requires the complex formulations of inks in which the electroactive matters are solubilized or dispersed. The stabilization of electronic inks is critical and generally requires the tedious and empirical formulation of mixtures of varied concentrations. Electronic inks made of Carbon Nanotubes (CNTs) have been widely explored. However, the conductivity of CNT films remains significantly lower than that of individual CNTs. ${ }^{2}$ Their poor dispersibility in most solvents, their ability to aggregate easily and the high contact resistance between CNTs are the main reasons for this deviation. In the past decades, one of the most effective and nondestructive way to enhance the dispersibility of CNT is the non-covalent modifications of the CNT surface with organic mediating molecules, ranging from low molecular weight molecules to supramolecular polymers. ${ }^{3-5}$ Nevertheless, insulating polymers are not desirable with respect to their low conductivity, because they annihilate the excellent electrical properties of CNTs by acting as interfacial resistance. ${ }^{6}$ Thus, conjugated polymers (CPs), such as poly(3-hexylthiophene) $)^{7,}{ }^{8}$, polypyrroles $^{9}$, polycarbazole ${ }^{5,10,11}$, polyfluorene ${ }^{11,12}$ and poly(m-phenylene vinylene $)^{13}$, provide alternative strategies to disentangle CNTs while preserving their electronic properties. The strength of $\pi-\pi$ interactions between CPs 
and CNT outmost walls was proven to be much stronger than the interaction between nonconjugated polymers like polystyrene and CNT based on theoretical molecular dynamics studies. ${ }^{14}$ Such an excellent interfacial binding is pivotal in determining the charge transfer of nanotube-based polymer electronic devices. ${ }^{15}$

Although the inter-tube van der Waals interactions is weakened by the wrapping of CPs, wrapped CNTs are difficult to be dispersed in most common solvents due to the poor solubility of CPs, particularly in water. Thus, a structure which consists of a conjugated rod-block and a soluble coil block was recently explored to improve the dispersibility of functionalized CNTs. ${ }^{3,6,7}$ These materials exhibit similar optical and electrical properties as the $\pi$-CPs. Zou et al. reported that the dispersibility of CNTs wrapped with P3HT-b-PS copolymer $(2.5 \mathrm{mg} / \mathrm{ml}$ for SWNTs and 3.0 $\mathrm{mg} / \mathrm{ml}$ for MWNTs) is more than two times higher to that of wrapped CNTs by P3HT homopolymer with a lower percolation threshold in chloroform. ${ }^{7,}{ }^{16}$ However, among the block copolymers investigated, little work has been devoted to water-soluble $\mathrm{CPs}^{17}$ and their ability to individualize SWNTs. Furthermore, the electrical performance (e.g. conductivity) of such CNTCPs materials, attributed to the assembly of CPs and dispersed CNTs, has not yet been investigated in depth.

In the present work, we propose a well-defined strategy to synthesize P3HT- $b$-PSS copolymer by adding sulfonated groups on the PS part of P3HT-b-PS through a controlled sulfonation reaction, which is much more simpler than the reported one in reference ${ }^{18}$. The presence of an ionic coil block (PSS) both enhances the electrical properties of the $\pi$-conjugated backbone (the so-called self-doped polymers) and leads to a good solubility in polar solvents. ${ }^{19}$ Thus the obtained P3HT$b$-(PS-stat-PSS) copolymer exhibits excellent solubility in water and superior conductivity $(\sim 1$ $\mathrm{S} / \mathrm{m})$ as compared to P3HT $\left(\sim 10^{-6} \mathrm{~S} / \mathrm{m}^{20}\right.$ or $\left.\sim 3.8 \times 10^{-3} \mathrm{~S} / \mathrm{m}^{21}\right)$ and P3HT-b-PS $\left(\sim 2 \times 10^{-5} \mathrm{~S} / \mathrm{m}\right.$ in 
this work or less than $10^{-7} \mathrm{~S} / \mathrm{m}^{7}$ ). Its formulation with SWNTs demonstrates its efficiency to debundle and disperse SWNTs in water. Individualization of SWNTs is demonstrated by photoluminescence in Raman spectra. The highest concentration obtained of stable SWNT dispersion is around $3.8 \mathrm{mg} / \mathrm{mL}$ using a 4:1 copolymer to SWNT mass ratio. This concentration is higher than that reported for P3HT-b-PS dispersed CNTs in organic solvents. ${ }^{7}$ In order to examine the stability of the P3HT- $b$-(PS-stat-PSS)/SWNT aqueous dispersions, we used a recent microfluidic tool to monitor in situ the concentration process of dilute dispersions up to completely dry SWCNTs/CPs films. ${ }^{22}$ Such experiments lead to the phase diagram for the SWNT/P3HT-b-(PS-stat-PSS) aqueous dispersion, which reveals a micro-phase concentration in a well-defined concentration range. The ultimate conductivity of the final dry composite achieved is as high as $370 \mathrm{~S} / \mathrm{m}$ which is four orders of magnitude higher than the case of SWNT/PS nanocomposite with SWNT dispersed by P3HT-b-PS ${ }^{7}$, although the percolation threshold observed for P3HT-b-(PS-stat-PSS) dispersed SWNTs nanocomposite approaches $15 \mathrm{vol} \%$.

\section{Experimental}

2.1 Synthesis of P3HT-b-(PS-stat-PSS).

Our strategy includes three main steps: (i) synthesis of $\omega$-functional P3HT as a macro-RAFT initiator, (ii) polymerization of styrene and (iii) controlled sulfonation of the PS block.

(1) Synthesis of P3HTmacroinitiator: Allyl terminated regioregular P3HT homopolymer was synthesized via a Nickel-catalyzed chain growth polymerization with the end groups functionalized using Grignard metathesis polymerization (GRIM) according to the literature procedure from McCullough et al. ${ }^{23}$ and Yokozawa et al. ${ }^{24}$. The allyl end group of the polymer was further treated with 9-borabicyclo(3.3.1)nonane (9-BBN) followed by the addition of 
hydrogen peroxide and aqueous $\mathrm{NaOH}$ solution resulting in a hydroxypropyl end group. ${ }^{25}$ Then, hydroxyl terminated P3HT $(0.3 \mathrm{~g}, 0.03 \mathrm{mmol})$ was dissolved in distilled dichloromethane at $40^{\circ} \mathrm{C}$. The mixture was stirred followed by addition of 3benzylsulfanylthiocarbonylsulfanylpropionic acid (RAFT agent) (0.041g, 0.15 mmol),4(dimethylamino) pyridinium-p-toluene sulfonate(DPTS) $(0.01 \mathrm{~g}, 0.03 \mathrm{mmol})$ and N,N'diisopropylcarbodiimide (DIPC) $(0.03 \mathrm{~mL}, 0.195 \mathrm{mmol})$ under nitrogen atmosphere. ${ }^{26}$ The reaction mixture was stirred for 3 days at $30^{\circ} \mathrm{C}$. The resulting P3HT macroinitiator was precipitated in methanol and filtered.

(2) RAFT polymerization ofP3HT-b-PS: P3HT macroinitiator ( $0.3 \mathrm{~g}, 0.03 \mathrm{mmol})$ was added in freshly distilled styrene $(4 \mathrm{~mL}, 34.8 \mathrm{mmol})$ under $\mathrm{N}_{2}$ protection. The reaction mixture was stirred at $45^{\circ} \mathrm{C}$ until P3HT macroinitiator was completely dissolved, and three freeze-pump-thaw cycles proceeded. Then, the reaction mixture was immersed in a thermostated oil bath at $120^{\circ} \mathrm{C}$ and the molecular weight of polystyrene was controlled by the time of reaction. Finally, the reaction was terminated by freezing the mixture in liquid $\mathrm{N}_{2}$. The resulting P3HT-b-PS copolymer was precipitated in methanol, filtered and dried in vacuum oven.

(3) Sulfonation of P3HT- $\boldsymbol{b}$-PS: P3HT-b-PS copolymer (1g, mol(styrene) $=8.26 \mathrm{mmol}$ ) was dissolved in $15 \mathrm{~mL}$ degassed dichloroethane under Ar atmosphere when a acetyl sulfate was prepared. Acetic anhydride $(1.6 \mathrm{~mL}, 16.52 \mathrm{mmol})$ was added in dichloroethane $(4 \mathrm{~mL})$, and the mixture was cooled down to $0^{\circ} \mathrm{C}$ followed by addition of sulfuric acid $(0.66 \mathrm{~mL}, 12.39 \mathrm{mmol})$ under Ar protection. The obtained acetyl sulfate was then added in the copolymer solution under $\mathrm{Ar}$ and the reaction mixture was heated to $60^{\circ} \mathrm{C}$ for 3 days. The resulting P3HT- $b$-(PS-stat-PSS) was withdrawn by filtration and dried. ${ }^{1} \mathrm{H}-\mathrm{NMR}$ spectrum (Bruker AVANCE I, 400MHz) and 
Size Exclusion Chromatography (SEC, PL-GPC 50 Plus (Agilent Technologies)) were used to indicate the composition and the dispersity of the copolymer.

\subsection{SWNT/copolymer dispersions}

Synthesized P3HT-b-(PS-stat-PSS) was dissolved in water by tip sonication treatment (Branson Sonifier S-250A equipped with a $13 \mathrm{~mm}$ step disruptor horn and a $3 \mathrm{~mm}$ tapered microtip) for 15 $\min$ at a frequency of $20 \mathrm{kHz}$, and centrifuged at $4000 \mathrm{rpm}$ for $30 \mathrm{~min}$ to separate extra copolymer pieces. Then the supernatant was used to disperse SWNTs at different concentrations using sonication during 90 min with the same frequency and cold water protection according to related experiments on similar materials. ${ }^{27,28}$ Raw SWNTs bundles were obtained from Unidym (Sunnyvale,CA, batch number R0513). These nanotubes are synthesized by a Chemical Vapor Deposition process called the HiPco process (High Pressure Carbon Monoxide). ${ }^{29}$ As-produced materials contain iron nanoparticles with a weight fraction of about $32.6 \mathrm{wt} \%$. SWNTs were used as received (in a form of a wet cake) without further purification or chemical modification. Such a protocol yields black, homogeneous nanotube dispersions.

2.3 Microfluidic investigation of the phase diagram of SWNT/copolymer dispersions and microfluidic printing of electrodes

We used two almost similar microfluidic devices to (i) investigate the phase diagram of the SWNT/copolymer dispersions, and (ii) to print electrodes starting from dilute dispersions. These devices both integrate a thin poly(dimethylsiloxane) PDMS membrane, across which water can permeate from a microchannel containing the dispersion, see Fig. 1a. Similar microfluidic devices were used recently to manufacture micro-scaled materials ${ }^{30,31}$, but also to investigate the phase diagrams of different complex fluids at the nanoliter scale. ${ }^{22,32}$ The detailed functioning of these devices can be found in the above cited references and is briefly summarized below. 
A microfluidic channel (typical dimensions $\mathrm{h}=5-30 \mu \mathrm{m}, \mathrm{w}=100 \mu \mathrm{m}$ ) is sealed by a thin PDMS membrane $(\mathrm{e}=35-60 \mu \mathrm{m})$ on a length $\mathrm{L}_{0}=10-15 \mathrm{~mm}$ (see Fig. 1a). Such devices are made using standard photolithography techniques: channels are made within a PDMS matrix and sealed by a glass slide. Pervaporation of water through the PDMS membrane (at a typical rate $\mathrm{qe}_{\mathrm{e}} \sim \mu \mathrm{m}^{2} / \mathrm{s}$ per unit length) drives a flow within the channel, see arrows in Fig. $1 \mathrm{~b}$ and c. We then used two different protocols depending on our applications.

(1) For the screening of the ternary phase diagram SWNT/copolymer/water, we monitor the shrinkage of a drop containing the dilute dispersions due to the water pervaporation along the channel (see Fig. 1b). Typical shrinkage kinetics are given by $\tau_{\mathrm{e}}=\mathrm{L}_{0} \mathrm{hw} /\left(\mathrm{L}_{\mathrm{oq}} \mathrm{e}\right) \approx 1500 \mathrm{~s}$. Details on the protocol to make such a drop (thanks to the gentle flow of an inert oil, Fluorinert FC40, in surrounding channels) can be found in reference ${ }^{22}$. In this particular regime, the pervaporationinduced flow within the drop prevents from the formation of concentration gradients during the drop's shrinkage (see ${ }^{22}$ for a complete demonstration of this result). Concentration within the drop thus follow $\phi(\mathrm{t}) \mathrm{L}(\mathrm{t})=\phi_{0} \mathrm{~L}_{0}$ where $\phi_{0}$ is the initial solute concentration (SWNT or copolymer) and $\mathrm{L}_{0}$ is the initial drop length $(15 \mathrm{~mm})$. We used a stereo-microscope (Olympus) coupled with a CCD camera to check for the possible de-mixing during the concentration of both SWNTs and copolymers, as shown in section 3.2. Such observations also reveal that no concentration gradients indeed develop during the shrinkage of the drop (revealed using the colored adsorption of the copolymer solution and/or the adsorption of the dispersed SWNT). Custom-made image analysis programs (Matlab) lead to precise estimates of the drop's length $\mathrm{L}(\mathrm{t})$ (accuracy $\pm 5 \mu \mathrm{m}$ ) and thus to precise estimates of the concentrations within the drop $\phi(\mathrm{t})$.

(2) For making electrodes, we proceeded as follows. The channel is connected to a reservoir (a simple hole punched in the PDMS matrix, $\sim 50 \mu \mathrm{L}$ ) containing the dilute complex fluid under 
study (SWNT/copolymer dispersion or polymer solutions, see section 3.2). The pervaporation of water along the channel drives a flow from the reservoir which concentrates slowly the nonvolatile solutes up to the tip of the channel, see Fig. 1c. After a transient time (typically $\sim 2 \mathrm{hrs}$ depending on the concentration and the geometrical parameters), a dense material starts to invade the channel, see Fig. 1c. The detailed mechanisms of such a nucleation and growth scenario were investigated in references ${ }^{30,32}$ for the case of nanoparticles, polymers, and surfactants. After the complete growth of a composite in the channel, the elastomeric mold is carefully peeled off from the substrate leaving thus printed microstructures on the substrate. Such a process works well for the cases investigated in the present work: pure PSS (Sigma-Aldrich, Mw 75 KDa, $18 \mathrm{wt} \%$ in water) and P3HT- $b$-(PS-stat-PSS) solutions and SWNT/P3HT-b-(PS-stat-PSS) dispersions leading to printed composites. This process makes also possible the printing of composites on flexible substrates as demonstrated in Fig. 1d, where we sealed the channels using another PDMS film deposited on a flexible PET film.

\subsection{Electrical characterization of the printed electrodes}

The conductivity of the electrodes printed by the method explained above was measured by impedance spectroscopy (7260 Impedance Analyzer, MaterialsMates Italia). For improving the measurements, the reservoir containing the solutions/dispersions is connected to multiple channels in parallel. We thus obtained up to 29 printed microstructures and we measured the conductivity of these parallel electrodes using silver paste deposited on each side of the strips, see in Fig. S1 in SI. To get values of conductivity, we also measured the widths and heights of the printed electrodes using mechanical profilometry (Veeco). Significant deviations from the channel dimensions were observed (e.g. $\mathrm{h}=30, \mathrm{w}=100 \mu \mathrm{m}$ leading to electrodes with $15 \times 90$ 
$\mu \mathrm{m}^{2}$ ). These deviations mainly come from the deformations of the PDMS matrix during the concentration process, and the corresponding mechanisms were investigated in ${ }^{31}$.

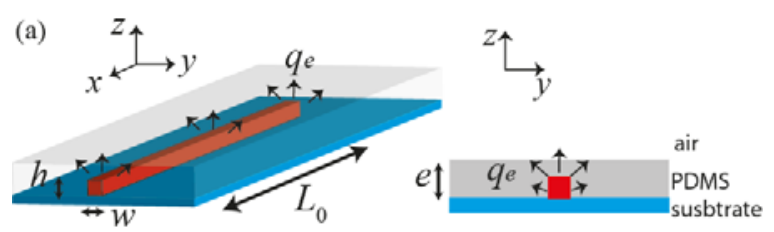

(b)

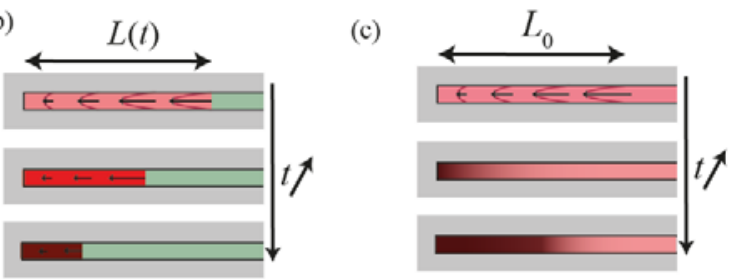

(d)

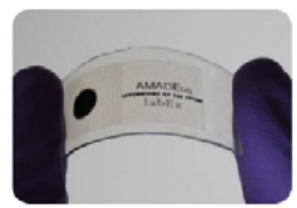

Fig 1.(a) 3D and top (schematic) views of microfluidic pervaporation. A microfluidic channel (typical dimensions $\mathrm{w}=100 \mu \mathrm{m}, \mathrm{h}=5-30 \mu \mathrm{m}$ and $\mathrm{L}_{0}=15 \mathrm{~mm}$ ) is embedded in a thin PDMS membrane $(\mathrm{e}=35-60 \mu \mathrm{m})$. Water pervaporates trough the PDMS membrane at a rate $\mathrm{q}_{\mathrm{e}}(\sim 1$ $\mu \mathrm{m}^{2} / \mathrm{s}$, per unit length). (b) The shrinkage of a drop due to the pervaporation of water makes possible the investigation of the ternary phase diagram SWNT/copolymer/water. Colors represent the different concentrations. The pervaporation-induced flow (see arrows) prevents from the development of concentration gradients within the drop. (c) Similar mechanisms lead to the formation of a dense printed composite when the channel is continuously connected to a reservoir containing the dilute SWNT/copolymer/water dispersion. Colors represent the different concentrations. (d) Picture of an array of printed electrodes on a flexible substrate made of a thin PMDS film deposited on a flexible substrate. The logo (Labex Amadeus) was printed using a standard printer on the PET sheet.

\section{Results and discussion}

3.1 Investigation of the synthesized copolymer 


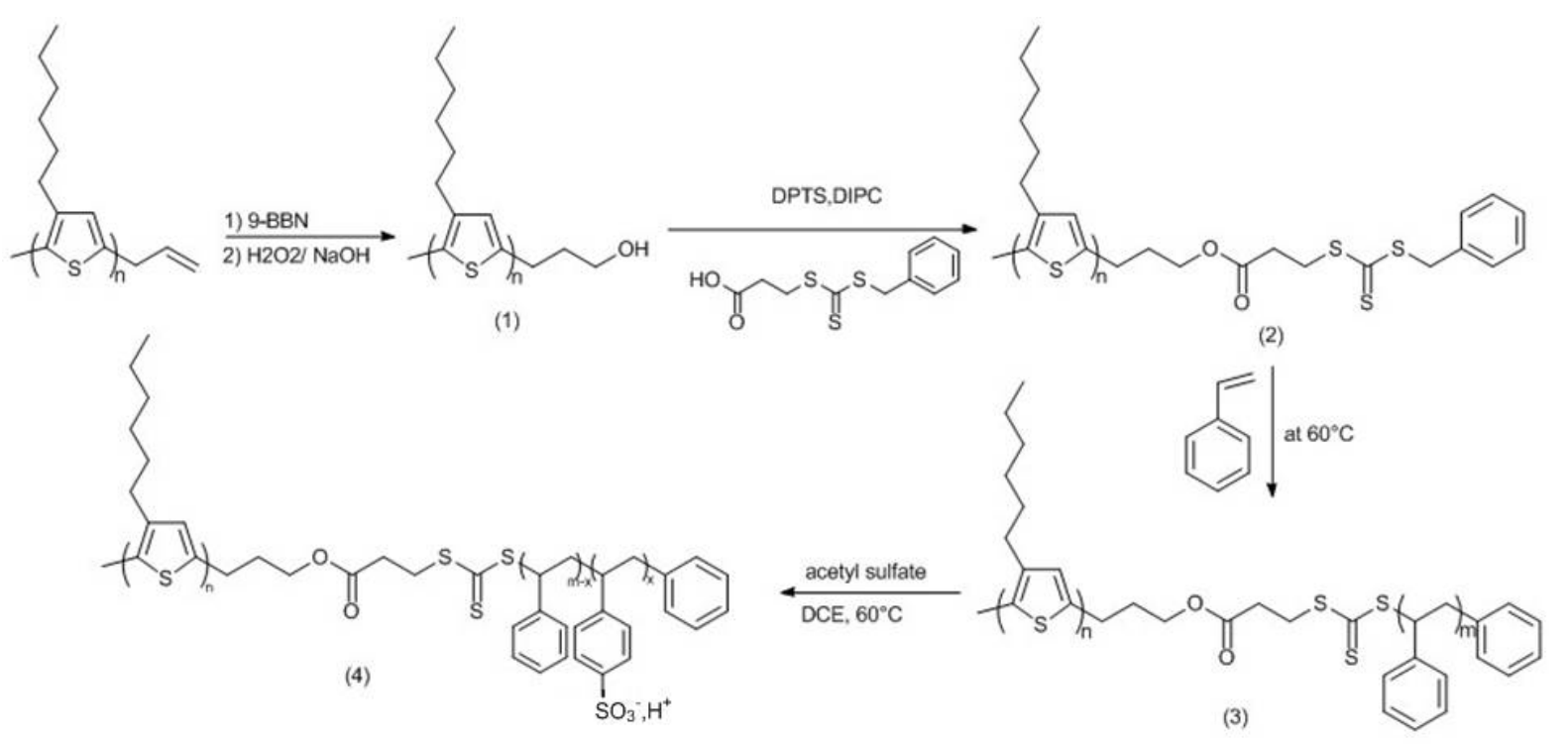

Scheme1. Synthetic processing of P3HT-b-(PS-stat-PSS) block copolymers

Scheme1 displays schematically the synthesis of P3HT-b-(PS-stat-PSS) copolymers. Regioregular P3HT homopolymer prepared from 2,5-dibromo-3-hexylthiophene via Nicatalyzed GRIM polymerization ${ }^{23}$ shows the degree of polymerization, $\mathrm{DP}_{\mathrm{n}}$ of $\sim 60$, and a number average molecular weight $\mathrm{Mn}$ of $10 \mathrm{KDa}$ with head-to-tail regioregularity of $94 \%$ estimated from ${ }^{1} \mathrm{H}$ NMR spectrum. SEC measurements indicate a low dispersity of $Đ \sim \mathrm{Mw} / \mathrm{Mn}=1.1$ (shown in Fig. S2). Then the allyl end group is further treated with 9-BBN followed by the addition of hydrogen peroxide and aqueous $\mathrm{NaOH}$ solution in order to obtain a hydroxypropyl end group (see 1 in scheme 1), which reacts with the RAFT agent to yield a $\omega$-thiocarbonate P3HT used as a macroinitiator for RAFT of PS (see 2 in scheme 1). The molecular weight of PS segments is strictly controlled by the reaction time of RAFT polymerization(see 3 in scheme 1). The achieved degree of polymerization ( $\mathrm{DP}_{\mathrm{n}}$ of $\sim 630$ and $\mathrm{Mn}$ of $65 \mathrm{kDa}$ ) has been calculated from the presence of phenyl ring protons at $6.58 \mathrm{ppm}$ in the ${ }^{1} \mathrm{H}$ NMR spectrum shown in Fig.2 (a). 


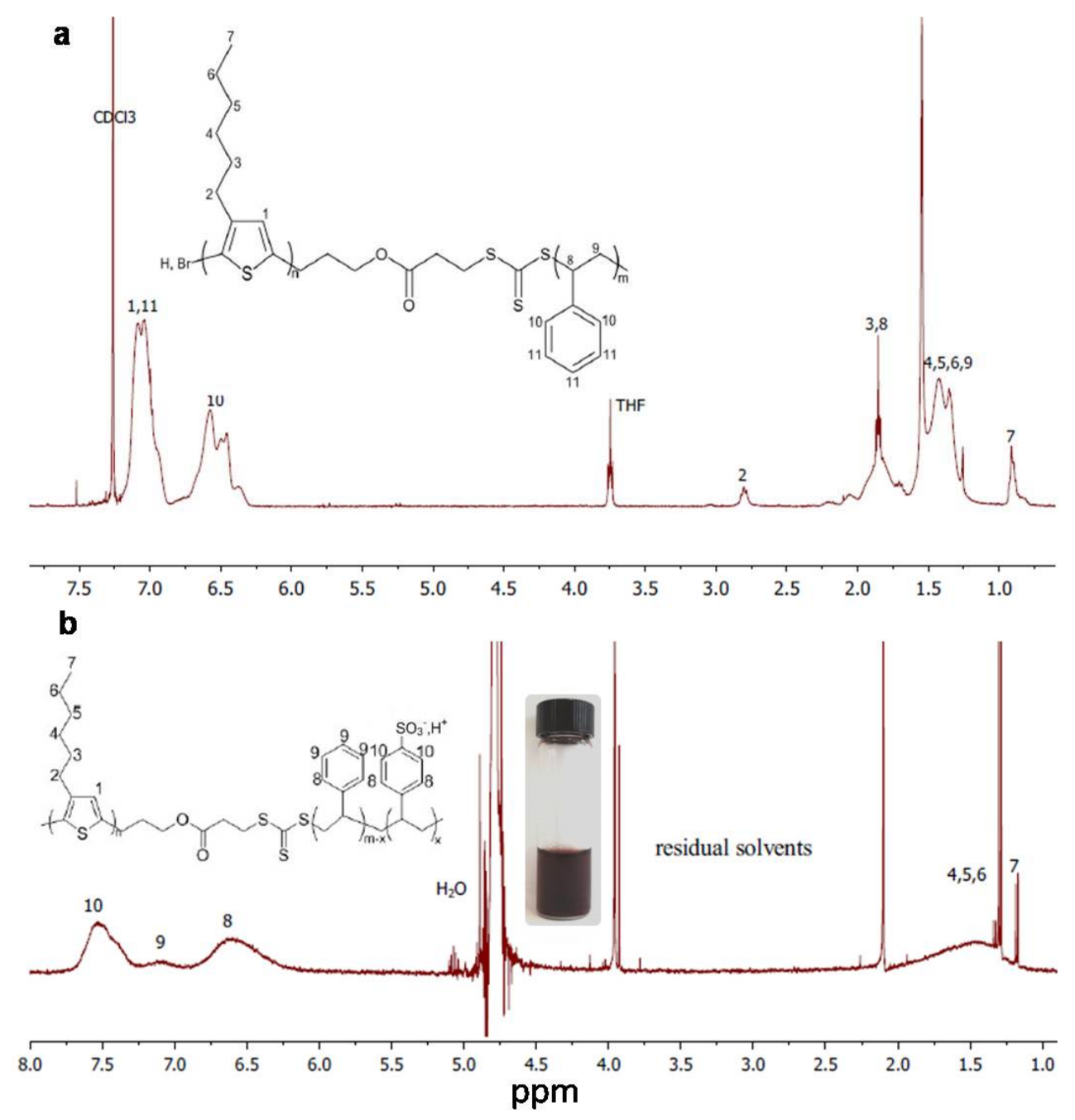

Figure 2. ${ }^{1} \mathrm{H}$ NMR spectra of (a) P3HT-b-PS and (b) P3HT-b-(PS-stat-PSS) block copolymer. The inset picture shows a copolymer/water solution in a vial.

Finally, the controlled sulfonation of the PS block is achieved through the addition of freshly prepared acetyl sulfate to P3HT- $b$-PS and degassed dichloroethane solution heated to $60^{\circ} \mathrm{C}$. The sulfonation level (SL) of styrene units is successfully controlled by the duration of the reaction, and the P3HT-b-(PS-stat-PSS) block copolymer is withdrawn by filtration (see 4 in scheme 1). It is noteworthy that such a sulfonation reaction does not induce detrimental effects on the copolymer structure, since the peaks corresponding to P3HT (between 1 and 2 ppm) and PS 
(7.10 and $6.62 \mathrm{ppm})$ are present in the spectra of the block copolymers (integrity of P3HT was checked on a separated sulfonation control reaction). The SL is determined by following the characteristic peak at $7.5 \mathrm{ppm}$ arising from the aromatic protons of the styrene sulfonate repeat units as shown in Fig.2 (b), which indicates a high SL value of $\sim 90 \%$. Thus, final copolymers can be defined as $\mathrm{P}_{3} \mathrm{HT}_{60}-b$-(PS63-stat-PSS 567$)$ according to its composition. Thanks to this high SL value, the resulting block copolymer exhibits a good solubility in water with the help of a gentle sonication treatment, as shown in Fig.2.

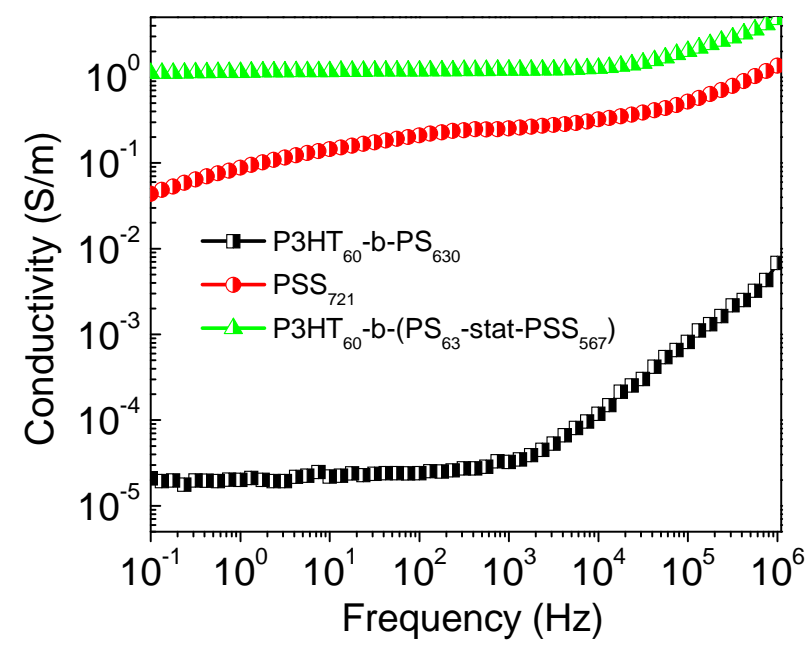

Figure 3.Frequency dependence of conductivity of P3HT- $b$-PS (obtained from Section 2.1(2)before sulfonation of the PS block), PSS (commercial product) and P3HT-b-(PS-statPSS).

To better understand the electrical features of our copolymer and to clarify their origin, the conductivity of each component in our copolymer was determined separately (see Fig. 3). We thus used the microfluidic technology detailed in Section 2.3(2) to print microstructured dry films on a glass substrate starting from aqueous solutions of the synthesized copolymer, of pure PSS and of P3HT-b-PS (see Fig. 1c). For these three cases, microfluidic pervaporation makes it possible to print homogeneous strips playing the role of electrodes and Fig. 3 displays their 
electrical conductivity as a function of frequency. Regioregular P3HT is a semi-conducting polymer which is broadly used as an electron donor in the active layer of solar cell with PCBM as electron acceptor. However, as expected from a non-doped semi-conducting p-type polymer, P3HT itself exhibits a relative low electrical conductivity, $\sim 10^{-6} \mathrm{~S} / \mathrm{m}$ in the observed frequency range of interest for most flexible electronic applications. ${ }^{20}$ The block copolymer P3HT- $b$-PS synthesized in this work, which has been reported to disperse CNTs in organic solvent ${ }^{7}$, shows a relatively higher conductivity of $\sim 2 \times 10^{-5} \mathrm{~S} / \mathrm{m}$ but still far from that of P3HT-b-(PS-stat-PSS), $\sim 1$ S/m. Meanwhile, commercial PSS presents a conductivity that compares with that of our copolymer. We thus conclude that the ionic conductivity of PSS segments dominates the resulting electrical property of the copolymer films in the investigated range of frequency. Furthermore, the conductivity of copolymers could be slightly varied by controlling the length of PSS segments, as shown in Fig.S3).

\subsection{Investigation of the SWNT/copolymer dispersions}

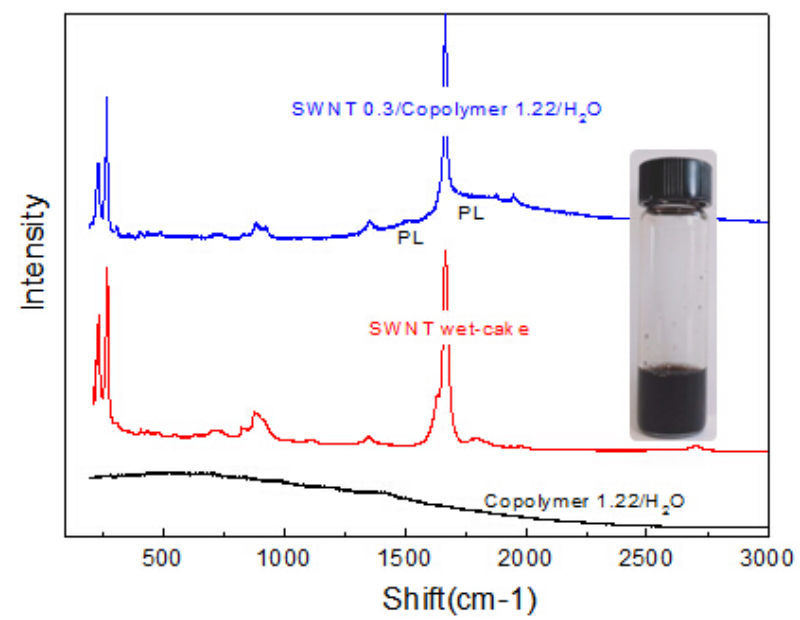

Figure 4. Raman spectra of a copolymer/water solution with $1.22 \mathrm{wt} \%$ copolymer, SWNT wetcake and a SWNT/copolymer dispersion with $0.3 \mathrm{wt} \%$ SWNT and $1.22 \mathrm{wt} \%$ copolymer in water. The incident laser wavelength is $785 \mathrm{~nm}$. The inset image shows the corresponding SWNT/copolymer dispersion in a vial. 
SWNTs are easily dispersed in water thanks to the presence of P3HT-b-(PS-stat-PSS) with typical tip sonication treatment. The stability of the SWNT/copolymer dispersions was also examined through a centrifugation test. After being subjected to a centrifugation treatment at $13200 \mathrm{rpm}$ for $30 \mathrm{~min}$, most SWNTs remained dispersed, and the highest SWNT concentration obtained in the supernatant is around $3.8 \mathrm{mg} / \mathrm{mL}$ with $4: 1$ copolymer to SWNT mass ratio. This concentration is much higher than the CNT dispersions with its analogue P3HT- $b$-PS in organic solvent ${ }^{7}$, indicating that our copolymer is an efficient dispersant for SWNTs in aqueous solutions. As mentioned earlier, such an efficient dispersion is attributed to the good solubility of the PSS block in water. Furthermore, as shown in Figure 4, the difference of intensity background in Raman spectra of dispersed nanotubes and wet-cake materials reveals unambiguously the presence of individualized semiconducting SWNTs in the SWNT/copolymer suspensions. Indeed, the observed difference, specified by PL in the spectra of Figure 4, corresponds to photoluminescence emission by semiconducting nanotubes with an excitation wavelength of $785 \mathrm{~nm}$. Photoluminescence of nanotubes is quenched when the particles are under the form of bundles. ${ }^{33}$ A detailed determination of the chirality of the dispersed and individualized nanotubes would require combined optical absorption measurements, Raman spectroscopy and photoluminescence mapping ${ }^{34}$, which is out of the scope of the present work. As shown in Fig. 4, the characteristic peaks of the suspension, radial breathing modes (RBM) at low frequencies between 250 and $350 \mathrm{~cm}^{-1}$, D and $\mathrm{G}^{\prime}$ bands around 1280 and $260 \mathrm{~cm}^{-1}$, and $\mathrm{G}$ bands in the range of $1550-1600 \mathrm{~cm}^{-1}$, appear indeed at the same wave number as that of nanotubes bundles in wet-cakes except the additional broad photoluminescence (PL) lines. ${ }^{27}$ The Raman spectrum of the copolymer solution is also presented in Fig.4 without any PL. All these 
investigations show that our synthesized copolymer is a promising candidate for dispersing SWNTs in aqueous solutions for its use in flexible electronics.

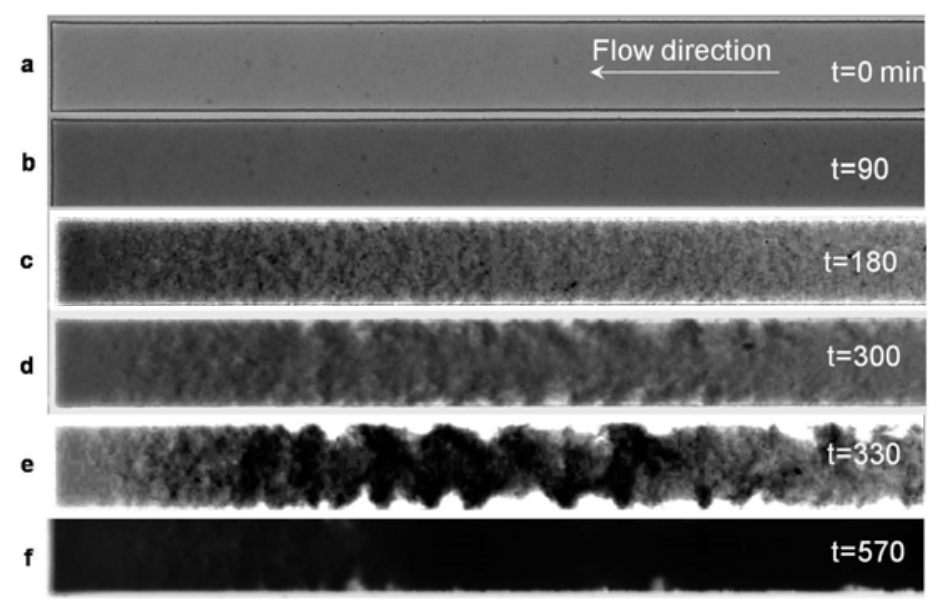

Figure 5. Series of snapshots obtained from the tip of a single channel in microevaporator during the concentration process of a SWNT/copolymer solution with initial concentration: $\phi \mathrm{SWNT}=0.02$ $\mathrm{vol} \%, \phi$ copolymer $=0.1 \mathrm{vol} \%$. The elapsed times are noted above. Contrasts of $\mathrm{e}, \mathrm{d}$ and $\mathrm{f}$ are increased to show the CNT aggregation. The channel width is $100 \mu \mathrm{m}$.

Fig. 5 shows the concentration kinetics of a SWNT/copolymer dispersion used as prepared in a microfluidic channel sealed by a PDMS membrane across which water pervaporates. This figure shows more precisely typical snapshots at the tip of the channel (the complete video can be found in SI-V1) during the continuous concentration of both CNTs and copolymers due to the pervaporation-induced flow (see Experimental Section). The channel appears initially transparent when filled with the dilute dispersion, and then becomes progressively more and more opaque due to the concentration increase of CNTs (Fig. 5a-c). The typical rate of concentration is both controlled by the time scale of this pervaporation process $\left(\tau_{\mathrm{e}} \sim 1500 \mathrm{~s}\right)$ and the initial concentration of the dispersion. At a well-defined time (Fig. 5d), aggregation of CNTs shows up with black strip patterns oriented perpendicular to the channel. These black strips keep progressing and accumulating towards the tip of channel (Fig. 5e-f). Finally, a dense and dry 
material invades progressively the whole channel towards the reservoir on a typical time scale $\sim 70 \mathrm{hrs}$. This process leads finally to printed electrodes within the channel, and we discuss later their electrical properties as a function of the SWNT concentrations. The above scenario is observed for dispersions with SWNT and copolymer volume ratio higher than 1:20, however for lower ratios (down to pure copolymer solutions), we do not observe any demixing during the pervaporation-induced drying of the solutions/dispersions.

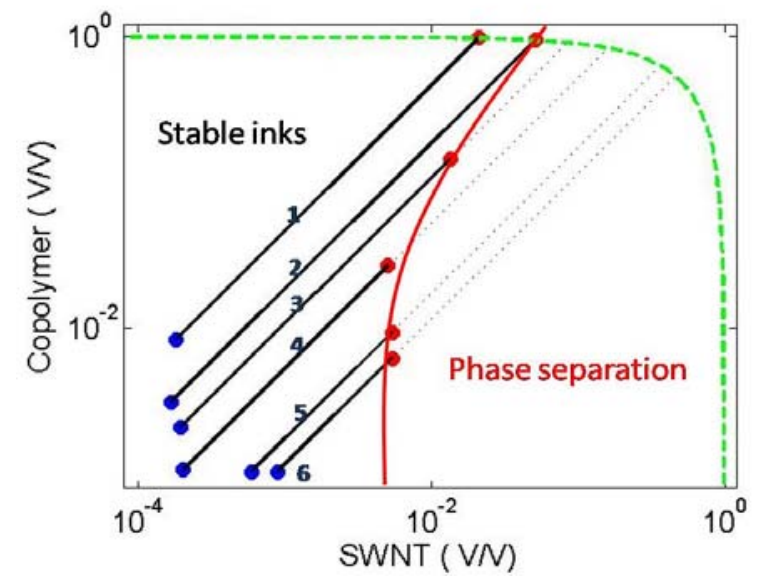

Figure 6. Phase diagram for SWNT/copolymer solution. Blue dots indicate initial concentration of the SWNTs in droplet, red dots that at phase separation and green dashed line that in final dry SWNT/copolymer composite from 0 to $100 \%$. The black lines and blue dotted ones in between are for guiding eyes.

Deeper insights into this microphase separation were investigated with the help of the microfluidic technique shown in Fig.1b and detailed in reference ${ }^{22}$. A droplet of a dilute dispersion at a given volume fractions of CNTs and copolymer is left to pervaporate within a long channel (length $15 \mathrm{~mm}$ ). The uniform pervaporation of water along the droplet across the thin PDMS membrane imposes a simple relation between the shrinkage of the drop length and the concentration of SWNTs and copolymer described by:

$\phi(\mathrm{t}) \mathrm{L}(\mathrm{t})=\phi(0) \mathrm{L}(0)$, 
where $\phi(0), \mathrm{L}(0)$ are the initial concentrations and the initial drop length respectively, and $\phi(\mathrm{t})$, $\mathrm{L}(\mathrm{t})$ that at time $\mathrm{t}$. More detailed information about the design of this microfluidic chip leading to homogeneous concentration during drying can be found in reference ${ }^{22}$. Thanks to this technique, we could quantitatively determine the concentrations of each component of the ternary phase diagram during the evaporation process. It should be noted that, a very thin layer of copolymer is deposited on the channel PDMS surface owing to the amphiphilic property of the copolymer, and this may induce uncertainties in the $5-10 \%$ range. Nevertheless, as shown in Fig. 6, a generalized phase diagram is obtained by detecting the demixing of samples with various initial concentrations of SWNTs and copolymer. Fig. S4 presents typical snapshots taken during the shrinkage of droplet (Fig. S4a), the demixing (zoom at the tip of channel, Fig. S4b), and the complete video can be found in SI-V2 (only the tip of channel is observed for sake of clarity). It is interesting to find that this demixing could not be observed for the samples with $\phi(C N T)<5$ vol\% (values estimated in the final dry composite shown as sample 2 in Fig. 6), thus a quite uniform composite film is obtained as shown in video in SI-V3 (only the tip of channel is observed for sake of clarity).

The detailed information about the concentration of each sample corresponding to the initial, the demixing and the final evaporation process are shown in Table S1. It is found that stable dispersions (sample 1 and 2) without demixing during the concentration are the samples with the higher copolymer concentrations. For sample 2, $31.2 \mathrm{mg}$ copolymer is used for dispersing1.68 mg SWNTs. At this concentration a sufficient amount of polymers is present to provide an effective stabilization of the nanotubes. The involved mechanisms of stabilization include presumably both steric and electrostatic interactions considering that the PSS groups are exposed towards the solvent. 


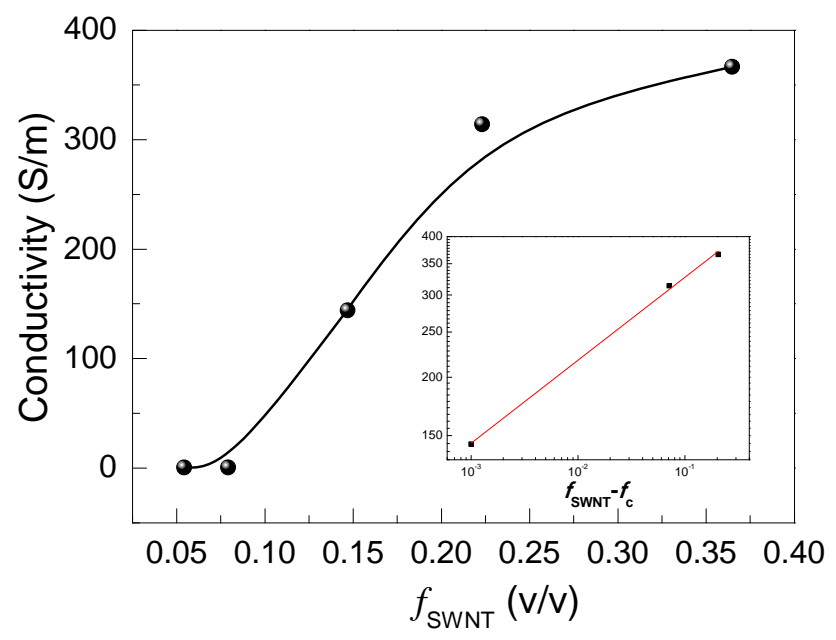

Figure 7. Conductivity as a function of SWNT concentration for SWNT/copolymer dry composite films

The large size of the adsorbed polymer chains are key advantages for efficient stabilization of SWNTs in water but it retards the percolation of the CNTs in dry films. The above argument is confirmed by the variation of the conductivity of the SWNT/copolymer composites with the SWNT concentration shown in Fig. 7. Note that continuous films could still be obtained from the samples despite the demixing leading to the aggregation of the CNTs. Although the samples with $\phi(\mathrm{CNT}) \leq 5$ vol $\%$ remains uniform during the concentration process, the corresponding conductivities are similar to that of the pure copolymer film $(\sim 1 \mathrm{~S} / \mathrm{m})$. A significant increase of the conductivity shows up around $15 \mathrm{vol} \%$, up to a very high conductivity of about $370 \mathrm{~S} / \mathrm{m}$ at $\phi(\mathrm{CNT})=36 \mathrm{vol} \%$. Such a behavior suggests a percolation threshold around $15 \mathrm{vol} \%$ with a critical exponent $\mathrm{t}=0.2$ according to $\sigma_{\mathrm{c}} \propto\left(f_{\mathrm{swNT}}-f_{\mathrm{c}}\right)^{\mathrm{t}}$, where $\sigma_{\mathrm{c}}$, $\mathrm{t}$ are the conductivity of SWNT/copolymer nanocomposites and the critical exponent in the conductive region respectively. Similar values were reported for MWNT/PVDF nanocomposites, however, with a rather low conductivity $\sim 10^{-4} \mathrm{~S} / \mathrm{m}$ at $1 \mathrm{~Hz} .{ }^{35}$ 
For such SWNT, the theoretical percolation threshold is expected to be much smaller because of their extreme high aspect ratio. However, the percolation threshold of SWNT-based composites depends both on the size distribution of the CNTs but also on other factors including interactions, correlation of orientations, waviness, and inter-tube tunneling distance between CNTs. ${ }^{36}$ This leads to two competing requirements: (i) the need for a good dispersant, and (ii) the smallest contact resistance between CNTs in the final composite. For very well dispersed CNTs in a insulating matrix, the conductivity is dominated by the matrix even for concentrations above the theoretical threshold, which may explain the low conductivity reported in the literature for CNTs composites $\left(f_{\mathrm{c}}=0.03 \mathrm{wt} \%\right.$ with $\sigma_{\mathrm{c}} \sim 10^{-3} \mathrm{~S} / \mathrm{m}^{7}, f_{\mathrm{c}}=0.095 \mathrm{wt} \%$ with $\left.\sigma \mathrm{c} \sim 10^{-4} \mathrm{~S} / \mathrm{m}^{37}\right)$. In our system, we do not observe any CNT aggregation during the drying process for concentration below $\phi(\mathrm{CNT}) \leq 5$ vol\%, leading to conductivities dominated by the copolymer matrix as explained above. However, we observe a huge increase of the conductivity suggesting the exposure of CNTs surface for $\phi(\mathrm{CNT})>5$ vol\%, which progressively becomes the main interactions between CNTs. We believe that this effect comes from the observed aggregation of CNTs during the drying process leading to the formation of CNTs-rich clusters embedded in a copolymer-rich matrix (see Fig. 4e). The percolation of these clusters may yield a very high conductivity as the contact resistances between CNTs are no more dominated by the copolymer matrix in the CNTrich cluster. Such a high conductivity of our system exhibits a strong potential for future electronics applications. From a more general point of view the microfluidic pervaporation method is shown to be a valuable tool to optimize the formulation of electronic inks using in particular environment friendly aqueous solvents and minute amounts of materials.

\section{CONCLUSIONS}


Amphiphilic P3HT-b-(PS-stat-PSS) block copolymer was synthesized through a simple and well-controlled method including GRIM polymerization of P3HT, RAFT polymerization of PS and sulfonation of PS. This route makes it possible to easily tune the size of each segment to adapt the solvent applied from nonpolar to polar. In this case, $\mathrm{P}_{3 \mathrm{HT}} 60-b-\left(\mathrm{PS}_{63}-\mathrm{stat}_{-} \mathrm{PSS}_{567}\right)$ with large PSS segments exhibits excellent solubility in water which is essential for CNT water-based dispersion. Microfluidic tools were then used to both investigate the phase diagram of the dispersions and make microstructured films playing the role of electrodes. Our block copolymer presents a promising high conductivity, $\sim 1 \mathrm{~S} / \mathrm{m}$, arising from the ionic conductivity of the dominant PSS segment. Furthermore, Raman spectroscopy revealed unambiguously the presence of individualized semiconducting SWNTs in the SWNT/copolymer dispersions which proves the efficiency of our copolymer at dispersing SWNTs. Demixing is observed during the concentration of the SWNT/copolymer dispersions with low mass ratio copolymer/CNT. This demixing promotes a high conductivity of $370 \mathrm{~S} / \mathrm{m}$ at high concentration of CNT, $\phi(\mathrm{CNT})=36 \mathrm{vol} \%$. The microfluidic pervaporation method is shown thereby to be a valuable tool for optimizing the formulation of electronic inks and for finding the most suitable compositions to achieve targeted properties. Our future work will be focused on the implementation of such nanocomposites made with SWNT/copolymer dispersions within electronic devices and on the characterization of their performances.

\section{ASSOCIATED CONTENT}

*Supporting Information

The Supporting Information is available free of charge on the ACS Publications website at DOI:

\section{AUTHOR INFORMATION}


Corresponding Authors: Dr. J-B. Salmon and Dr. E. Cloutet

* E-mail: jean-baptiste.salmon-exterieur@solvay.com; cloutet@enscbp.fr

The authors declare no competing financial interest.

\section{ACKNOWLEDGMENTS}

This work was supported by the LabEx AMADEus (ANR-10-LABX-42) in the framework of IdEx Bordeaux (ANR-10-IDEX-03-02) i.e. the Investissements d'Avenir programme of the French government managed by the Agence Nationale de la Recherche. Authors are grateful to Dr. J. Leng and future Dr. C. Laval for microfluidic chips design and Matlab code, and Dr. J. Jolly for Raman investigation.

\section{- REFERENCES}

(1. Vosgueritchian, M.; Tok, J. B. H.; Bao, Z., Nature Photonics 2013, 7 (10), 769-771; Kaltenbrunner, M.; Sekitani, T.; Reeder, J.; Yokota, T.; Kuribara, K.; Tokuhara, T.; Drack, M.; Schwoediauer, R.; Graz, I.; Bauer-Gogonea, S.; Bauer, S.; Someya, T., Nature 2013, 499 (7459), 458-+; Baeg, K.-J.; Caironi, M.; Noh, Y.-Y., Advanced Materials 2013, 25 (31), 4210-4244.

2. $\quad$ De Volder, M. F. L.; Tawfick, S. H.; Baughman, R. H.; Hart, A. J., Science 2013, 339 (6119), 535539.

3. Kim, S. W.; Kim, T.; Kim, Y. S.; Choi, H. S.; Lim, H. J.; Yang, S. J.; Park, C. R., Carbon 2012, 50 (1), 333.

4. Gerstel, P.; Klumpp, S.; Hennrich, F.; Altintas, O.; Eaton, T. R.; Mayor, M.; Barner-Kowollik, C.; Kappes, M. M., Polymer Chemistry 2012, 3 (8), 1966-1970; Berton, N.; Lemasson, F.; Tittmann, J.; Stuerzl, N.; Hennrich, F.; Kappes, M. M.; Mayor, M., Chemistry of Materials 2011, 23 (8), 2237-2249; Nish, A.; Hwang, J.-Y.; Doig, J.; Nicholas, R. J., Nature Nanotechnology 2007, 2 (10), 640-646; Nish, A.; Hwang, J.-Y.; Doig, J.; Nicholas, R. J., Nanotechnology 2008, 19 (9); Hwang, J.-Y.; Nish, A.; Doig, J.; Douven, S.; Chen, C.W.; Chen, L.-C.; Nicholas, R. J., Journal of the American Chemical Society 2008, 130 (11), 3543-3553; Imin, P.; Imit, M.; Adronov, A., Macromolecules 2012, 45 (12), 5045-5050; Imin, P.; Imit, M.; Adronov, A., Macromolecules 2011, 44 (23), 9138-9145; Imin, P.; Cheng, F.; Adronov, A., Polymer Chemistry 2011, 2 (6), 1404-1408.

5. Rice, N. A.; Adronov, A., Macromolecules 2013, 46 (10), 3850-3860.

6. Park, H. S.; Choi, B. G.; Hong, W. H.; Jang, S.-Y., Journal of Physical Chemistry C 2012, 116 (14), 7962-7967.

7. Zou, J.; Liu, L.; Chen, H.; Khondaker, S. I.; McCullough, R. D.; Huo, Q.; Zhai, L., Advanced Materials 2008, 20 (11), 2055-+.

8. Giulianini, M.; Waclawik, E. R.; Bell, J. M.; De Crescenzi, M.; Castrucci, P.; Scarselli, M.; Motta, N., Applied Physics Letters 2009, 95 (1); Goh, R. G. S.; Motta, N.; Bell, J. M.; Waclawik, E. R., Applied Physics Letters 2006, 88 (5); Schuettfort, T.; Snaith, H. J.; Nish, A.; Nicholas, R. J., Nanotechnology 2010, 21 (2). 
9. Liu, X. L.; Ly, J.; Han, S.; Zhang, D. H.; Requicha, A.; Thompson, M. E.; Zhou, C. W., Advanced Materials 2005, 17 (22), 2727-+.

10. Lemasson, F. A.; Strunk, T.; Gerstel, P.; Hennrich, F.; Lebedkin, S.; Barner-Kowollik, C.; Wenzel, W.; Kappes, M. M.; Mayor, M., Journal of the American Chemical Society 2011, 133 (4), 652-655.

11. Lemasson, F.; Berton, N.; Tittmann, J.; Hennrich, F.; Kappes, M. M.; Mayor, M., Macromolecules 2012, 45 (2), 713-722.

12. Ozawa, H.; Ide, N.; Fujigaya, T.; Niidome, Y.; Nakashima, N., Chemistry Letters 2011, 40 (3), 239241.

13. Star, A.; Stoddart, J. F.; Steuerman, D.; Diehl, M.; Boukai, A.; Wong, E. W.; Yang, X.; Chung, S. W.; Choi, H.; Heath, J. R., Angewandte Chemie-International Edition 2001, 40 (9), 1721-1725.

14. Yang, M. J.; Koutsos, V.; Zaiser, M., Journal of Physical Chemistry B 2005, 109 (20), 10009-10014. 15. Lo, K. C.; Li, S. Y.; Chan, W. K., Journal of Materials Chemistry C 2014, 2 (37), 7739-7751; Luo, Y. Q.; Santos, F. A.; Wagner, T. W.; Tsoi, E.; Zhang, S. J., Journal of Physical Chemistry B 2014, 118 (22), 6038-6046.

16. Zou, J.; Chen, H.; Chunder, A.; Yu, Y.; Huo, Q.; Zhai, L., Advanced Materials 2008, 20 (17), 3337-+. 17. Kang, Y. K.; Lee, O.-S.; Deria, P.; Kim, S. H.; Park, T.-H.; Bonnell, D. A.; Saven, J. G.; Therien, M. J., Nano Letters 2009, 9 (4), 1414-1418.

18. Erothu, H.; Kolomanska, J.; Johnston, P.; Schumann, S.; Deribew, D.; Toolan, D. T. W.; Gregori, A.; Dagron-Lartigau, C.; Portale, G.; Bras, W.; Arnold, T.; Distler, A.; Hiorns, R. C.; Mokarian-Tabari, P.; Collins, T. W.; Howse, J. R.; Topham, P. D., Macromolecules 2015, 48 (7), 2107-2117.

19. Jiang, H.; Taranekar, P.; Reynolds, J. R.; Schanze, K. S., Angewandte Chemie-International Edition 2009, 48 (24), 4300-4316.

20. Obrzut, J.; Page, K. A., Physical Review B 2009, 80 (19).

21. Nicholson, P. G.; Ruiz, V.; Macpherson, J. V.; Unwin, P. R., Physical Chemistry Chemical Physics 2006, 8 (43), 5096-5105.

22. Ziane, N.; Guirardel, M.; Leng, J.; Salmon, J.-B., Soft Matter 2015, 11 (18), 3637-3642.

23. Jeffries-El, M.; Sauve, G.; McCullough, R. D., Advanced Materials 2004, 16 (12), 1017-+; JeffriesEl, M.; Sauve, G.; McCullough, R. D., Macromolecules 2005, 38 (25), 10346-10352; Sheina, E. E.; Liu, J. S.; lovu, M. C.; Laird, D. W.; McCullough, R. D., Macromolecules 2004, 37 (10), 3526-3528.

24. Miyakoshi, R.; Yokoyama, A.; Yokozawa, T., Journal of the American Chemical Society 2005, 127 (49), 17542-17547; Yokoyama, A.; Miyakoshi, R.; Yokozawa, T., Macromolecules 2004, 37 (4), 1169-1171. 25. Iovu, M. C.; Jeffries-El, M.; Sheina, E. E.; Cooper, J. R.; McCullough, R. D., Polymer 2005, 46 (19), 8582-8586.

26. Stenzel, M. H.; Davis, T. P.; Fane, A. G., Journal of Materials Chemistry 2003, 13 (9), 2090-2097.

27. Zamora-Ledezma, C.; Buisson, L.; Moulton, S. E.; Wallace, G.; Zakri, C.; Blanc, C.; Anglaret, E.; Poulin, P., Langmuir 2013, 29 (32), 10247-10253.

28. Badaire, S.; Poulin, P.; Maugey, M.; Zakri, C., Langmuir 2004, 20 (24), 10367-10370.

29. Nikolaev, P.; Bronikowski, M. J.; Bradley, R. K.; Rohmund, F.; Colbert, D. T.; Smith, K. A.; Smalley, R. E., Chemical Physics Letters 1999, 313 (1-2), 91-97.

30. Merlin, A.; Salmon, J.-B.; Leng, J., Soft Matter 2012, 8 (13), 3526-3537; Angly, J.; lazzolino, A.;

Salmon, J.-B.; Leng, J.; Chandran, S. P.; Ponsinet, V.; Desert, A.; Le Beulze, A.; Mornet, S.; Treguer-

Delapierre, M.; Correa-Duarte, M. A., Acs Nano 2013, 7 (8), 6465-6477.

31. Demko, M. T.; Cheng, J. C.; Pisano, A. P., Acs Nano 2012, 6 (8), 6890-6896.

32. Daubersies, L.; Leng, J.; Salmon, J.-B., Lab on a Chip 2013, 13 (5), 910-919.

33. O'Connell, M. J.; Bachilo, S. M.; Huffman, C. B.; Moore, V. C.; Strano, M. S.; Haroz, E. H.; Rialon, K. L.; Boul, P. J.; Noon, W. H.; Kittrell, C.; Ma, J. P.; Hauge, R. H.; Weisman, R. B.; Smalley, R. E., Science 2002, 297 (5581), 593-596. 
34. Dresselhaus, M. S.; Dresselhaus, G.; Saito, R.; Jorio, A., Physics Reports-Review Section of Physics Letters 2005, 409 (2), 47-99; lakoubovskii, K.; Minami, N.; Kazaoui, S.; Ueno, T.; Miyata, Y.; Yanagi, K.; Kataura, H.; Ohshima, S.; Saito, T., Journal of Physical Chemistry B 2006, 110 (35), 17420-17424.

35. Yuan, J.-K.; Yao, S.-H.; Dang, Z.-M.; Sylvestre, A.; Genestoux, M.; Bai, J., Journal of Physical Chemistry C 2011, 115 (13), 5515-5521.

36. Zakri, C.; Poulin, P., Journal of Materials Chemistry 2006, 16 (42), 4095-4098; Kyrylyuk, A. V.; van der Schoot, P., Proceedings of the National Academy of Sciences of the United States of America 2008, 105 (24), 8221-8226; Li, C.; Thostenson, E. T.; Chou, T.-W., Composites Science and Technology 2008, 68 (6), 1445-1452; Ma, Y.; Cheung, W.; Wei, D.; Bogozi, A.; Chiu, P. L.; Wang, L.; Pontoriero, F.; Mendelsohn, R.; He, H., Acs Nano 2008, 2 (6), 1197-1204; Rahman, R.; Servati, P., Nanotechnology 2012, 23 (5);

Schilling, T.; Jungblut, S.; Miller, M. A., Physical Review Letters 2007, 98 (10); Vigolo, B.; Coulon, C.;

Maugey, M.; Zakri, C.; Poulin, P., Science 2005, 309 (5736), 920-923; Yu, Y.; Song, G.; Sun, L., Journal of Applied Physics 2010, 108 (8).

37. Yan, Y.; Cui, J.; Poetschke, P.; Voit, B., Carbon 2010, 48 (9), 2603-2612. 


\section{Synthesis of conductive copolymer and phase diagram of its suspension with SWNT for printing by microfluidic technology}

Shenghong Yao, ${ }^{\dagger},{ }^{\ddagger} \S$ Aikaterini Bethani, ${ }^{\dagger}$ Nadia Ziane, ${ }^{\ddagger}$ Cyril Brochon, ${ }^{\dagger}$ Guillaume Fleury, Georges Hadziioannou, ${ }^{\dagger}$ Philippe Poulin, ${ }^{\S}$ Jean-Baptiste Salmon, ${ }^{\ddagger,}$ Eric Cloutet, ${ }^{\dagger, *}$

${ }^{\dagger}$ Laboratoire de Chimie des PolymèresOrganiques (LCPO), CNRS (UMR5629), ENSCPB, 16 avenue PeyBerland, 33607 Pessac Cedex, France

${ }^{\ddagger}$ Laboratoire du Futur (LOF), CNRS (UMR5258), Université de Bordeaux, 178 Avenue du Docteur Schweitzer, 33608 Pessac Cedex, France

${ }^{\S}$ Centre de Recherche Paul Pascal(CRPP), Université de Bordeaux, 115 avenue Schweitzer, 33600 Pessac Cedex, France
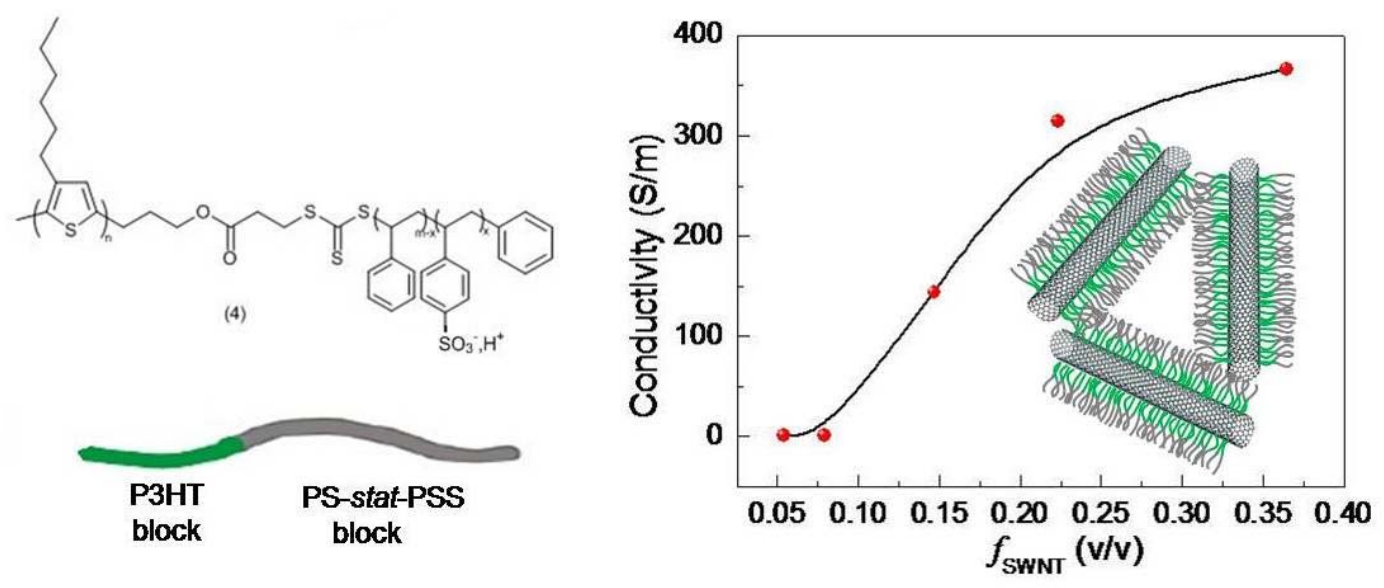